\title{
Saphirine et staurotide riche en magnésium et chrome dans les amphibolites et anorthosites à corindon du Vohibory Sud, Madagascar
}

\author{
par ChRISTIAN NICOLLET
}

C.U.R. de Tuléar, Madagascar et Institut de Géologie, 5, rue Kessler, 63000 Clermont-Ferrand, France.

Résumé. - Les amphibolites à corindon, saphirine ou gédrite et parfois grenat du Vohibory dérivent du métamorphisme de leucotroctolites dans les conditions intermédiaires entre le faciès amphibolite de haut degré et le faciès granulite de haute pression $\left(\mathrm{Pt} \simeq 9-11,5 \mathrm{kbar} ; \mathrm{T}=750^{\circ}-800^{\circ} \mathrm{C}\right)$. La présence de saphirine ou gédrite est liée aux variations du rapport $\mathrm{SiO}_{2} /(\mathrm{FeO}+\mathrm{MgO})$ de la roche ; celui-ci est fonction de la proportion $\mathrm{Ol} / \mathrm{Pl}$ et de la teneur en anorthite du plagioclase de la roche magmatique. L'association homblende-corindon exclut gédrite = saphirine dans les roches à plagioclase selon la réaction hb $+\mathrm{co} \rightleftharpoons \mathrm{gd}+\mathrm{sa}+\mathrm{pl}+\mathrm{H}_{2} \mathrm{O}$. Un banc d'anorthosite à corindon, saphirine et grenat (Py35 Alm50 Gr13) contient une staurotide riche en magnésium $(\mathrm{XMg}=52)$ et en chrome $\left(\mathrm{Cr}_{2} \mathrm{O}_{3}=0,8-2,2 \%\right)$. D'une manière générale, la staurotide magnésienne est rare car limitée à des métasédiments ou métasabites peralumineux, pauvres en silice et de rapport $\mathrm{Mg} / \mathrm{Fe}$ élevé. De plus, la présence de sodium semble favoriser l'association gédrite-hornblende $(+$ corindon) aux dépens de staurotide-saphirine $(+$ anorthite). Pour les températures considérées, la limite inférieure de stabilité en pression de la staurotide magnésienne $(\mathrm{XMg} \sim 50)$ est proche de la transition disthène-sillimanite.

Mots-clés : saphirine, staurotide $(\mathrm{Mg}, \mathrm{Cr})$, gédrite, amphibolite, anorthosite, granulite, Vohibory-Madagascar.

Sapphirine and Mr-, Cr-rich staurolite in corundum-bearing amphibolite and anorthosite from Vohibory, Madagascar.

Abstract. - Corundum, sapphirine or gedrite, and locally garnet occur in amphibolites derived from leucotroctolites during regional Barrovian metamorphism. The latter reached conditions transitional between amphibolite facies and granulite facies at high pressure, estimated to be $\mathrm{Pt}=9-11.5 \mathrm{kbar}, \mathrm{T}=750-800^{\circ} \mathrm{C}$. The occurrence of sapphirine or gedrite depends on the $\mathrm{SiO}_{2} /(\mathrm{FeO}+\mathrm{MgO})$ ratio and on anorthite content of the primary plagioclase. The hornblende - corundum assemblage precludes gedrite + sapphirine in plagioclasebearing rocks according to the reaction $\mathrm{hb}+\mathrm{co} \rightleftharpoons \mathrm{gd}+\mathrm{sa}+\mathrm{pl}+\mathrm{H}_{2} \mathrm{O}$. An anorthosite vein with corundum, garnet and sapphirine contains $\mathrm{Mg}-, \mathrm{Cr}$-rich staurolite $\left(\mathrm{XMg}^{2}=52 ; \mathrm{Cr}_{2} \mathrm{O}_{3}: 0.8-2.2 \mathrm{wt}\right.$. \%). $\mathrm{Mg}$-rich staurolite is unusual because restricted to peraluminous, silica-deficient rocks with high $\mathrm{Mg} / \mathrm{Fe}$ ratio. Moreover the presence of sodium favours gedrite-hornblende-corundum assemblages instead of staurolite-sapphirine-plagioclase. For the temperatures considered, the low pressure stability limit of magnesian staurolite $(\mathrm{XMg} \sim 50)$ is close to the kyanite-sillimanite transition.

Key words : sapphirine, staurolite (Mg-, Cr-rich), gedrite, amphibolite, anorthosite, granulite, VohiboryMadagascar.

\section{INTRODUCTION}

La saphirine se forme dans un large domaine de pression et de température à la limite entre le faciès amphibolite profond et le faciès granulite (Seifert, 1974 ; Ackermand et al., 1975). La relative rareté de ce minéral dans la nature ne tient donc pas à des conditions de stabilité restreintes mais au fait que la saphirine ne peut apparaître que dans des roches de composition chimique très limitée : il s'agit de métasédiments magnésiens constitués presque exclusivement de $\mathrm{MgO}-\mathrm{Al}_{2} \mathrm{O}_{3}-\mathrm{SiO}_{2}$ (Windley et al., 1984); ou bien de roches magmatiques associées à des complexes intrusifs basiques-ultrabasiques (Sills et al., 1983). Ce dernier type de roche contient également $\mathrm{CaO}$. Cette différence notable dans le chimisme des deux types de roche-hôte va influencer la nature des phases minérales associées à la saphirine. Ainsi le feldspath est généralement absent dans les métasédiments tandis que les roches orthodérivées sont souvent des amphibolites.

Dans le secteur étudié, de telles amphibolites à saphirine et corindon sont associées à un banc d'anorthosite qui contient une staurotide riche en magnésium (que nous nommerons "staurotide magnésienne" dans la suite de cet article) avec un rapport XMg atteignant 52. Des staurotides magnésiennes ont été récemment signalées dans un -schiste, en Antarctique (XMg $=40-42$, Grew et Sandiford, 1984), dans des granulites à saphi- 
rine, dans les Alpes centrales $(X M g=34-43$, Droop et Bucher-Nurminen, 1984) et en Afrique du Sud $(X M g=49$, Schreyer et al., 1984), dans une éclogite, en Norvège $(\mathrm{XMg}=50$, Smith, 1984) et dans une métatroctolite en Nouvelle Zélande (XMg=43-56, Ward, 1984). Dans tous ces cas, à l'exception de la staurotide magnésienne de l'anorthosite du Vohibory, ce minéral n'est pas en équilibre avec les autres minéraux des roches-hôtes. Les roches susceptibles de contenir de la staurotide magnésienne ont des compositions voisines de celles dans lesquelles se forme la saphirine.

\section{CONTEXTE GÉOLOGIQUE}

La formation précambrienne du Vohibory (Figure 1) est affectée par un métamorphisme de pression intermédiaire de type barrovien (Boulanger, 1954). Le métamorphisme est progressif depuis le faciès schiste vert (extrémité NW du secteur) jusqu'au faciès granulite de haute pression à hornblende (sur la bordure Est). Les amphibolites à saphirine et corindon sont systématiquement associées à des serpentinites, des amphibolites à grenat et des granulites à grenat (salite + almandin + magnésiohornblende + andésine) (Nicollet, 1983). Trois gisements sont actuellement connus (Figure 1). A Anavoha, on distingue :

- des amphibolites à saphirine ou gédrite, corindon et grenat,

- des amphibolites sans saphirine ni gédrite, - un banc métrique d'anorthosite à corindon et grenat,

Un massif de métatroctolite coronitique est associé à ces roches.

A Marolinta et à Ianapera, le grenat est absent dans les amphibolites à saphirine ou gédrite et corindon.

\section{ETUDE PÉTROGRAPHIQUE DES AMPHIBOLITES À CORINDON ET ROCHES ASSOCIÉES}

\section{Abréviations utilisées}

ab : albite, amp : amphibole, an : anorthite, anth : anthophyllite, ch : chlorite, co : corindon, $\mathrm{cpx}$ : clinopyroxène, di : disthène, ga : grenat,

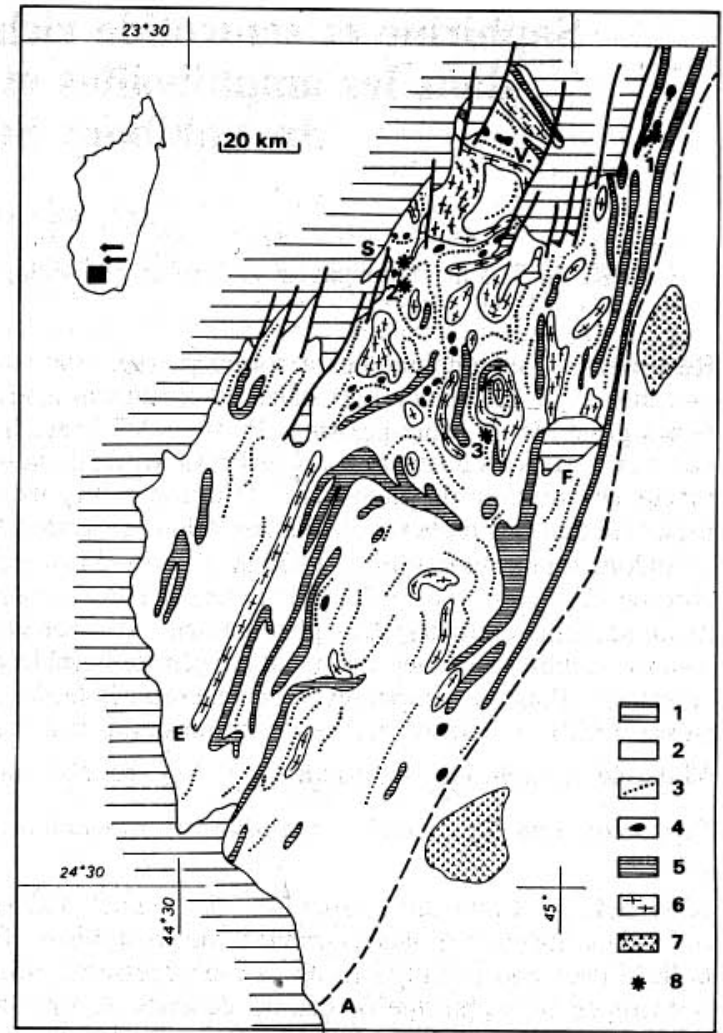

FIG. 1. - Carte simplifiée de la formation métamorphique du Vohibory. (Les nombreux filons basaltiques crétacés qui traversent la région (Boulanger, 1957 ; Nicollet, 1983) ne sont pas représentés). (1) formations sédimentaires, (2) gneiss migmatitiques, (3) principaux bancs d'amphibolites, (4) serpentinites, (5) calcaires métamorphiques, (6) orthogneiss, (7) anorthosites, (8) amphibolites à saphirine et corindon : 1 - Ianapera, 2 - Marolinta, 3 - Anavoha ; tirets : limite entre les formations du Vohibory et d'Ampanihy. V : Mont Vohibory, S : ancienne mine de charbon de la Sakoa ; A, E, F : villes d'Ampanihy, Ejeda et Fotadrevo. (En encart les flèches indiquent l'origine approximative des saphirines décrites par Lacroix (1929)).

Sketch map of the Precambrian metamorphic rocks of the Vohibory area.

gd : gédrite, hbv : hornblende verte, ma : margarite, ol : olivine, opx : orthopyroxène, pl : plagioclase, sa : saphirine, si : sillimanite, spv, spb : spinelle vert, brun, $\mathrm{Mg}$ ou Fe std : staurotide, pôle magnésien ou ferrifère, zo : zoïsite, ss : solution solide, $\mathrm{V}$ : vapeur, $\mathrm{XMg}=\mathrm{Mg}^{++}$ $\times 100 / \mathrm{Mg}^{++}+\mathrm{Fe}^{++}, \mathrm{Pt}$ : pression totale. 
La métatroctolite associée aux amphibolites d'Anavoha est une roche à grain fin présentant un litage magmatique grossier; elle contient de rares lentilles décimétriques d'anorthosite. Le plagioclase avec $72 \%$ du pôle anorthite est plus sodique que le plagioclase normatif. Quelques cristaux d'augite bordés d'amphibole brune montrent une texture ophitique relictuelle. Le plus souvent, les minéraux ferromagnésiens constituent un ensemble coronitique qui comprend depuis le centre jusqu'à la périphérie (Figure 2) :

- un agrégat polycristallin granuleux de bronzite ;

- une auréole médiane de diopside et/ou pargasite ;

- une auréole de grenat et/ou une auréole externe symplectitique de spinelle vert et pargasite.

On observe exceptionnellement de l'olivine au coeur des amas d'orthopyroxène. Ces minéraux sont magnésiens. En particulier, la composition du grenat exprimée en pôles purs est : $50 \% \mathrm{Py}$, $32 \%$ Alm-Spe, $17 \%$ Gros.

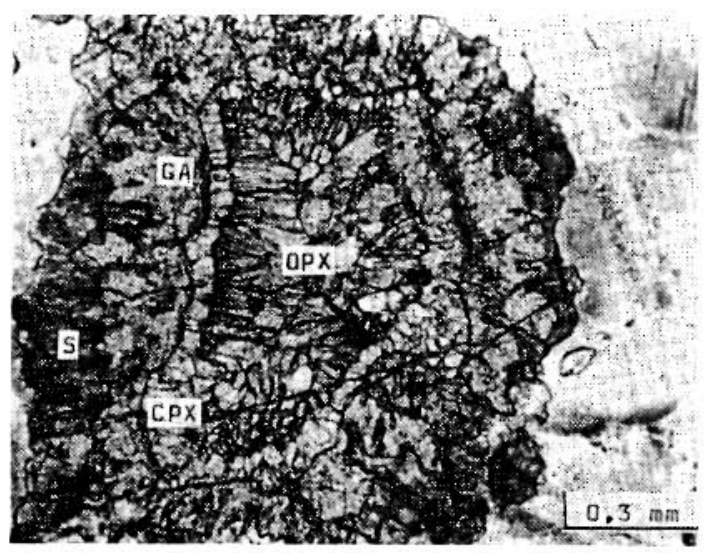

FIG. 2. - Auréole réactionnelle complexe dans une métatroctolite d'Anavoha composée, du centre vers la périphérie, par : - un amas d'orthopyroxènes granuleux. - une auréole médiane de clinopyroxènes, - une auréole de grenat, - une auréole symplectitique $(S)$ de spinelle vert et d'amphibole verte : échantillon A4. L.N.

Complex reaction coronas within metatroctolite from Anavoha. Central mosaic of granular orthopyroxene is successively surrounded by a middle corona of clinopyroxenes, a garnet corona and a symplectitic corona $(S)$ composed of green spinel and green amphibole ; sample A4; plane polarized light.
La transition est brutale entre la métatroctolite et les amphibolites à saphirine ou gédrite. La minéralogie de ces roches est variée et on peut définir les paragenèses suivantes :

(1) $\mathrm{gd}+\mathrm{hbv}$

(2) $\mathrm{gd}+\mathrm{hbv}+\mathrm{pl}+\mathrm{co}$

(3) $\mathrm{gd}+\mathrm{hbv}+\mathrm{pl}+\mathrm{co}+\mathrm{ga}$

(4) $h b v+p l+c o+g a+s a \pm s p v$

L'orthoamphibole et la saphirine n'existent pas ensemble dans une même paragenèse. Exceptionnellement, quelques cristaux de saphirine ont été rencontrés dans une amphibolite à deux amphiboles (échantillon 54b). La proportion des amphiboles avec le plagioclase est très variable et on passe progressivement à une anorthosite. Le grenat n'est pas présent systématiquement. Il présente des habitus et des dimensions variables : en grains millimétriques, disposés en chapelet ; en cristaux en atoll (Figures 4 et 5) ou en cristaux demi-centimétriques et automorphes. Les deux premiers habitus témoignent d'une évolution coronitique. La saphirine forme des tablettes allongées à l'intérieur du plagioclase (Figure 3) ou de la hornblende (Figure 4). Elle englobe souvent du corindon et/ou du spinelle vert. Le spinelle vert est rare dans les roches à orthoamphibole. Le spinelle brun est exceptionnel dans les amphibolites.

Les amphibolites à saphirine ou gédrite. passent à des amphibolites à gros grain dans lesquels ces deux minéraux sont absents. Signalons l'existence de disthène et staurotide en inclusions dans un grenat décimétrique. Le corindon est entouré d'une couronne de spinelle vert sombre et d'une couronne de plagioclase. Cet assemblage $\mathrm{Spv}+\mathrm{pl}$ va remplacer complètement le corindon. L'association minéralogique est : $\mathrm{hbv}+\mathrm{pl}+\mathrm{ga}+\mathrm{co} \pm \mathrm{spv} \pm$ minéraux opaques.

Le passage est progressif entre l'anorthosite à grenat et corindon et les amphibolites à corindon. Des tablettes de corindon et du grenat sont irrégulièrement répartis dans l'anorthosite. A côté de ces minéraux centimétriques, des minéraux millimétriques peuvent être présents en petite quantité $(<5 \%)$. Il s'agit des deux amphiboles, de staurotide, de spinelle brun, plus rarement de saphirine, rutile et chlorite ; du disthène n'existe qu'en inclusion dans le grenat, associé ou non avec de la staurotide et du plagioclase. La staurotide forme des cristaux squelettiques de quelques millimètres de long, asso- 


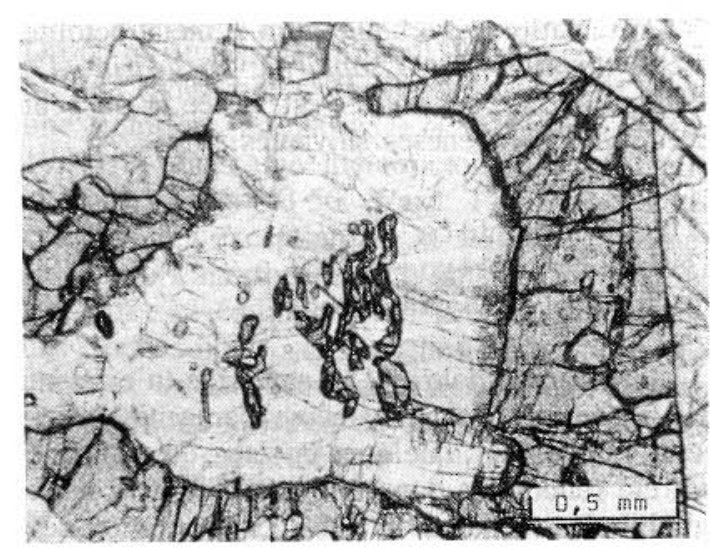

FIG. 3. - Saphirine incluse dans un plagioclase entouré par un grenat en atoll, automorphe à l'extrados. Echantillon A7. L.N.

Sapphirine included plagioclase rimmed by garnet. Sample A7. ; plane polarized light.

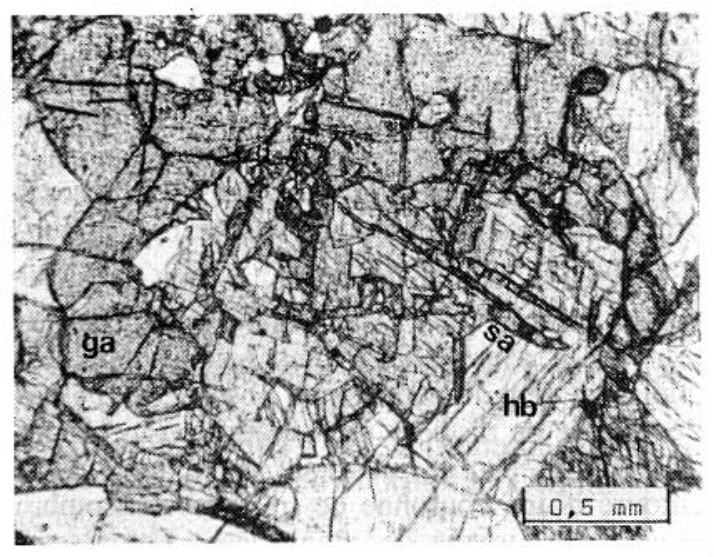

FIG. 4. - Tablettes de saphirine dans une hornblende entourée par un grenat en atoll. Echantillon 52. L.N. Sapphirine platelets in hornblende rimmed by garnet. Sample 52 ; plane polarized light.

ciés à du plagioclase et parfois du spinelle brun et de la saphirine (Figure 5). Elle est pléochroïque : brun jaune selon $\mathrm{Ng}$, jaune clair selon $\mathrm{Np}$ et vert pâle selon $\mathrm{Nm}$. La saphirine, très rare dans cette roche, forme de très petits cristaux (quelques dizièmes de millimètres) isolés dans le plagioclase ou associés à du spinelle brun ou encore aux agrégats à staurotide+plagioclase + spinelle brun (Figure 5). Elle n'est pas directement associée au corindon comme dans les amphibolites. Quelques rares petits cristaux de rutile sont isolés dans le plagioclase ou associés au spinelle brun. De la chlorite (tardive ?) jalonne des fissures dans la staurotide et la saphirine (Figure 5). La roche est hétérogène même à l'échelle d'une lame mince. On remarque en particulier qu'une augmentation de la proportion de la staurotide s'accompagne d'une diminution de celle des amphiboles. Il semble que l'on puisse définir les paragenèses suivantes :

(2') $\mathrm{Pl}+\mathrm{co} \pm \mathrm{gd} \pm \mathrm{hbv}$

(3') $\mathrm{Pl}+\mathrm{ga}+\mathrm{co} \pm \mathrm{gd} \pm \mathrm{hbv}$

(5) $\mathrm{Pl}+\mathrm{ga}+\mathrm{co} \pm \mathrm{std} \pm \mathrm{amph}(?) \pm \mathrm{spb}$

(6) $\mathrm{Pl}+\mathrm{ga}+\mathrm{co} \pm \mathrm{std} \pm \mathrm{sa} \pm \mathrm{spb}$

(7) $\mathrm{Ga} \pm$ di \pm std $\pm \mathrm{pl}$

A Marolinta et Ianapera, on remarque l'absence de grenat dans les amphibolites à corindon. Les paragenèses sont :

(2) et (8) hbv $+\mathrm{pl}+\mathrm{co}+\mathrm{sa}+\mathrm{spv}$.

\section{COMPOSITION DES MINÉRAUX DES AMPHIBOLITES ET ANORTHOSITES}

Tous les minéraux des amphibolites et de l'anorthosite ont des compositions très homogènes. Les zonations sont faibles, par exemple dans les grenats, ou absentes. Les minéraux mafiques ont des rapports XMg élevés. Toutefois, ces rapports diminuent lorsque la proportion plagioclase/amphiboles augmente. Ils sont plus élevés à Marolinta qu'à Anavoha. De plus, dans l'anorthosite, les minéraux mafiques, à l'exception du grenat, sont chromifères.

\section{Amphiboles :}

Dans la classification de Leake (1978), l'amphibole vert pâle se range parmi les magnésiohornblendes tschermackitiques (Tableau I). Elles sont moins siliceuses et moins sodiques que les pargasites des auréoles coronitiques de la troctolite. Le rapport $\mathrm{XMg}$ est élevé : il varie entre 75 dans l'anorthosite, 85 dans les amphibolites les plus pauvres en plagioclase d'Anavoha et 88 dans les amphibolites de Marolinta.

L'orthoamphibolite est une gédrite selon la classification de Leake (1978). Toutefois, les teneurs élevées en magnésium et relativement faibles en alumine suggèrent qu'il s'agit d'une orthoamphibole intermédiaire entre les gédrites et les anthophyllites (Robinson et al., 1971). 


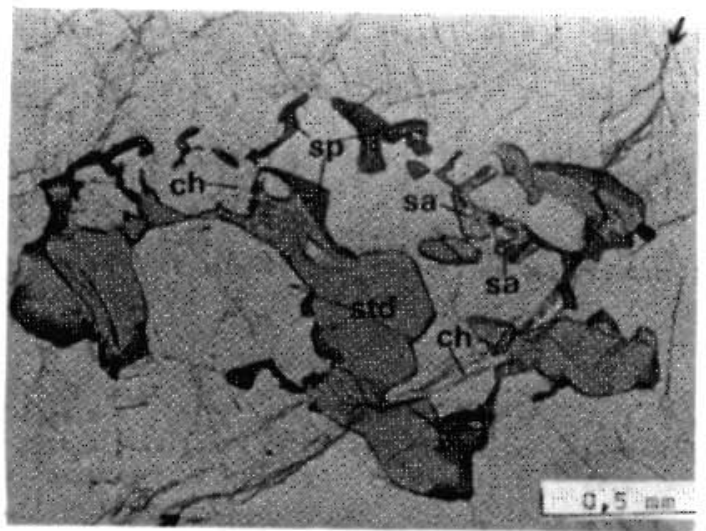

Fig. 5. - Staurotide squelettique associée à de la saphirine et partiellement frangée par du spinelle chromifere dans l'anorthosite d'Anavoha. La chlorite se localise préférentiellement le long d'une fracture (entre les flèches). Echantillon A24. L.N.

Skeletal staurolite associated with sapphirine and partly rimmed by chromian spinel. Anorthosite from Anavoha. Chlorite is preferentially located along a fracture (between the amows). Sample A24; plane polarized light.

Une diffractométrie aux rayons $\mathrm{X}$ montre en effet que cette amphibole est constituée de deux phases. Les paramètres du réseau cristallin : anthophyllite
gédrite
a $\quad 18,50 \pm 0,02 \AA$
$18,54 \pm 0,02 \AA$
b $\quad 17,96 \pm 0,03 \AA$
c $\quad 5,25 \pm 0,01 \AA$
$17,77 \pm 0,02 \AA$
$5,26 \pm 0,01 \AA$

démontrent bien l'existence de fines exsolutions (invisibles au microscope) d'anthophyllite dans la gédrite. $\mathrm{XMg}$ varie entre 73 dans I'anorthosite et 82 dans les amphibolites sans plagioclase d'Anavoha tandis qu'il atteint 83 à Marolinta. Ces valeurs sont voisines de celles des amphiboles calciques. Le coefficient de distribution dans les couples hornblende - gédrite $(\mathrm{Kd}=\mathrm{Mg} /$ $\mathrm{Fe}(\mathrm{Hb}) / \mathrm{Mg} / \mathrm{Fe}(\mathrm{Gd}))$ est légèrement supérieur à l'unité (Figure 6) : ceci suggère que les conditions d'équilibre sont atteintes entre ces deux minéraux. On remarque également que le couple pargasite - orthopyroxène des couronnes internes dans une métatroctolite coronitique (éch. AI) montre des valeurs semblables de ce coefficient de distribution (Figure 6).

\section{Plagioclase :}

Il est calcique (anorthite). Il est plus basique que le plagioclase normatif.

\section{Grenat :}

Les grenats des amphibolites sont très magnésiens. Leurs compositions exprimées en pôles purs varient d'un échantillon à l'autre entre $53 \mathrm{et}$ $60 \% \mathrm{Py}$, avec $30-35 \%$ Alm et $7-13 \%$ Gros. Le grenat de l'anorthosite est nettement plus ferrifere avec $50 \%$ Alm et $35 \%$ Py (Tableau I). La répartition du magnésium et du fer entre hornblende et grenat est relativement homogène dans les amphibolites $(\mathrm{Kd}=2,6-3,5$ : figure 6). Ces valeurs sont similaires à celles observées entre la pargasite et le grenat des couronnes externes de métatroctolites. Le couple hb-ga de l'anorthosite, par les fortes teneurs en fer du grenat, présente un $\mathrm{Kd}$ légèrement supérieur $(=4,14)$.

\section{Spinelles :}

Le spinelle vert des amphibolites à saphirine est une solution solide de spinelle et hercynite identique à celle des métatroctolites. $\mathrm{XMg}$ est compris entre 65 et 71 . Le spinelle brun, rare dans les amphibolites, est chromifere (éch. 54b : $\mathrm{Cr}_{2} \mathrm{O}_{3}=19 \%$, tableau I). Dans l'anorthosite, le spinelle brun ne contient que $9 \%$ de $\mathrm{Cr}_{2} \mathrm{O}_{3}$. Dans les deux types de roche, le rapport $\mathrm{XMg}$ est égal à 48 (Tableau I). Le zinc n'a pas été dosé.

\section{Saphirine :}

Dans le système $\mathrm{SiO}_{2}-(\mathrm{Mg}, \mathrm{Fe}) \mathrm{O}-(\mathrm{Al}, \mathrm{Fe}$, Cr) $)_{2} \mathrm{O}_{3}$, les saphirines se localisent sur la ligne de substitution $\mathrm{R}^{2+}+\mathrm{Si} \rightleftharpoons 2 \mathrm{R}^{3+}$ : elles montrent une faible substitution au voisinage de la composition 7-9-3 (Figure 7). La saphirine de l'anorthosite se situe à droite de cette composition, dans le domaine des saphirines peralumineuses considérées comme métastables (Schreyer et Abraham, 1975). Les teneurs en $\mathrm{Fe}^{3+}$, calculées selon la méthode de Higgins et al. (1979) sont très faibles $\left(\mathrm{Fe}^{3+}<0,01\right)$. Le chrome, inférieur à $1 \%$ dans les amphibolites, atteint $3 \%$ dans la saphirine de l'anorthosite, valeur relativement élevée : dans le Massif Central français, Braud (1981) signale une saphirine avec $5,7 \%$ de $\mathrm{Cr}_{2} \mathrm{O}_{3}$; dans le massif de Fiskenaesset, Herd (1973) et Friend (1982) indiquent des teneurs de 


\begin{tabular}{|c|c|c|c|c|c|c|c|c|c|c|c|}
\hline $\begin{array}{l}\text { Echontilion } \\
\text { mingrol }\end{array}$ & 940 & $\begin{array}{c}A 7(4) \\
00\end{array}$ & A24 & $\begin{array}{l}340 \\
\text { So }\end{array}$ & A7 & $\begin{array}{l}\text { A24 } \\
\text { So }\end{array}$ & $\begin{array}{l}A 24 \\
s+4\end{array}$ & SAb & A7 & $\begin{array}{l}\text { A24 } \\
\text { SD }\end{array}$ & $\begin{array}{l}\mathrm{A24} \\
\mathrm{Ch}\end{array}$ \\
\hline $\mathrm{SIO}_{2}$ & 40,30 & 41,10 & 39,30 & 13.63 & 14.40 & 12,30 & 27,90 & & & & 28,92 \\
\hline $\mathrm{Al}_{2} \mathrm{O}$ & 22,50 & 23,04 & 22,40 & 63,90 & 63.40 & 63,40 & 53,80 & 46,96 & 66,18 & 96,80 & 21.02 \\
\hline$f \circ \delta^{3}$ & 18,30 & 15,49 & 24,00 & 4,61 & 4,10 & 6,05 & 9,10 & 22,48 & 14,27 & 23.40 & 7,53 \\
\hline $\operatorname{MnO}$ & & & 1,00 & & & & & & & & \\
\hline $\mathrm{MgO}_{\mathrm{g}}$ & 14,80 & 16,39 & 9,40 & 17.76 & 18,20 & 16,30 & 5,40 & 11,64 & $.19,34$ & 12,20 & 24,19 \\
\hline$c 00$ & 3,78 & 3,09 & 5,00 & & & & & & & & 1,34 \\
\hline $\mathrm{Na}_{2} \mathrm{O}^{\mathrm{O}}$ & & & & & & & & & & & 0.09 \\
\hline $\mathrm{TiO}_{2}^{2}$. & & & & & & & 1.00 & & & & 0,52 \\
\hline $\mathrm{Cr}_{2} \mathrm{O}_{3}$ & & & & & & 2,77 & 2.20 & 19,04 & & 8,30 & \\
\hline Totai & 99,60 & 99.91 & 101,30 & 99,92 & 100,10 & 100,82 & 99,40 & 100,12 & 99,79 & 100,70 & 83,61 \\
\hline \multicolumn{12}{|l|}{ Nombre } \\
\hline dexygane & 24 & & & 20 & & & 46 & 32 & & & 28 \\
\hline si & 5,98 & 6,00 & 5.97 & 1,60 & 1,68 & 1,45 & 7,53 & & & & 5,80 \\
\hline Al & 3,93 & 3,96 & 3.99 & 8,83 & 8,72 & 8,83 & 17,08 & 12,50 & 15,82 & 14.48 & 4,97 \\
\hline$F \bullet$ & 2,27 & 1,87 & 3,03 & 0,45 & 0,40 & 0,60 & 2,04 & 4,25 & 2,42 & 4.23 & 1.26 \\
\hline mn & & & 0,13 & & & & & & & & \\
\hline Mg & 3.27 & 3,36 & 2,12 & 3,11 & 3,16 & 2,87 & 2,19 & 3.92 & 5,85 & 3,93 & 7,23 \\
\hline$c_{0}$ & 0.60 & 0,61 & 0.81 & & & & & & & & 0,29 \\
\hline No & & & & & & & & & & & \\
\hline$x$ & & & & & & & & & & & 0.02 \\
\hline TI & & & & & & & 0,20 & & & & 0.00 \\
\hline $\mathrm{cr}$ & & & & & & 0.26 & 0.47 & 3,39 & & 1,42 & \\
\hline$\Sigma \cot \theta$ & 16,05 & 16.00 & 16.05 & 13,99 & 13,96 & 14,01 & 29,51 & 24.06 & 24.09 & 24,05 & 19,65 \\
\hline$x \sim g$ & 59 & 66 & 41 & 87 & 89 & 83 & 32 & 48 & 11 & 48 & 83 \\
\hline Py & 53 & 59 & 35 & & & & & & & & \\
\hline Aln & 37 & 31 & 90 & & & & & & & & \\
\hline or & 10 & 10 & 13 & & & & & & & & \\
\hline So & & & 2 & & & & & & & & \\
\hline
\end{tabular}

\begin{tabular}{|c|c|c|c|c|c|c|c|c|c|c|}
\hline \multirow{2}{*}{$\begin{array}{l}\text { Echontlilion } \\
\text { minbral }\end{array}$} & \multicolumn{2}{|c|}{$A 14(1)$} & \multicolumn{2}{|c|}{$900(2)$} & \multicolumn{2}{|c|}{$540(3)$} & \multicolumn{2}{|c|}{$124(31-3-6-7)$} & \multirow{2}{*}{$\begin{array}{l}54 b \\
P 1\end{array}$} & \multirow{2}{*}{$\begin{array}{l}\text { A24 } \\
P 1\end{array}$} \\
\hline & Ho & Od & Mo & od & Mb & od & HD & Od & & \\
\hline $\mathrm{SIO}_{2}$ & 47,30 & 48,36 & 46,73 & 45,70 & 44,80 & 44,68 & 43.90 & 43,43 & 45,75 & 46,72 \\
\hline $\mathrm{Al}_{2} \mathrm{O}_{3}$ & 11.97 & 12.86 & 15.65 & 19,06 & 15,97 & 18,74 & 16,02 & 19.17 & 35,02 & 35,87 \\
\hline$F \cdot 0^{3}$ & 5,45 & 9,00 & 4.63 & 7,97 & 7,13 & 10,81 & 8,35 & 12,53 & & \\
\hline mo & 0,06 & 0,12 & & & & 0.25 & & & & \\
\hline$M_{g} O$ & 17.67 & 23.35 & 16,91 & 22.07 & 15.53 & 21.26 & 14,53 & 19,58 & & \\
\hline $\mathrm{C} \bullet \mathrm{O}$ & 11,70 & 0,84 & 12,21 & 0,74 & 11,29 & 0.72 & 10,95 & 0.36 & 18.65 & 17,92 \\
\hline $\mathrm{Na}_{2} \mathrm{O}$ & 1.35 & 1,25 & & & & 0,50 & & 0.30 & 0.80 & 1,24 \\
\hline $\mathrm{K}_{2} \mathrm{O}$ & 0,06 & & & & & & 0.23 & & & \\
\hline $\mathrm{TiO}_{2}$ & 0,12 & 0,06 & & & 0,18 & & 0,94 & 0,45 & & \\
\hline $\mathrm{Cr}_{2} \mathrm{O}_{3}$ & 0.10 & 0,10 & & & & & 0,77 & 0,32 & & \\
\hline Totol & 93.78 & 95,94 & 96,13 & 95,54 & 94,90 & 96.96 & 95,69 & 96.54 & 100,22 & 101,75 \\
\hline \multicolumn{11}{|l|}{ Nombre } \\
\hline doxygane & 23 & & & & & & & & 32 & \\
\hline si & 6.76 & 6.77 & 6,58 & 6,35 & 6,47 & 6.24 & 6.35 & 6.14 & 8,40 & 8,43 \\
\hline AI & 2,02 & 2,12 & 2.60 & 3,12 & 2,72 & 3,09 & 2,73 & 3.20 & 7,59 & 7,63 \\
\hline Fo & 0,65 & 1,09 & 0.55 & 0,93 & 0,86 & 1,26 & 1,01 & 1.48 & & \\
\hline mn & 0,01 & 0,01 & & & & 0,03 & & & & \\
\hline $\mathrm{Mg}$ & 3,76 & 4,87 & 3.55 & 4.37 & 3.34 & 4,43 & 3,13 & 4,13 & & \\
\hline co & 1,79 & 0,13 & 1,84 & 0.11 & 1.75 & 0.11 & 1,70 & 0,08 & 3,67 & 3,47 \\
\hline No & 0,37 & 0,34 & & & & 0,13 & & 0.14 & 0,29 & 0,43 \\
\hline$\kappa$ & 0,01 & & & & & & 0,04 & & & \\
\hline$T 1$ & 0,01 & 0,01 & & & 0,02 & & 0,10 & 0.05 & & \\
\hline cr & 0,01 & 0,01 & & & & & 0,09 & 0,04 & & \\
\hline$\Sigma$ cot. & 15,40 & 15,32 & 15,12 & 15,00 & 15.16 & 15,29 & 15,15 & 15,26 & 19,95 & 19,26 \\
\hline$x \mathrm{Mg}$ & OS & 82 & 88 & 83 & 80 & 78 & 76 & 74 & & \\
\hline An & & & & & & & & & 93 & 89 \\
\hline
\end{tabular}




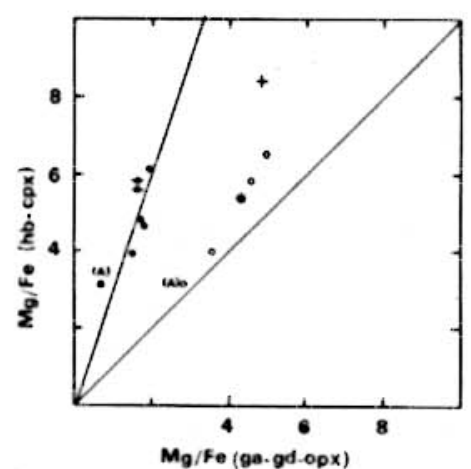

FIG. 6. - Répartition magnésium-fer des couples: opx-cpx (+) et opx-hb (*) dans les métatroctolites, hb-gd (o) dans les amphibolites à corindon et dans l'anorthosite d'Anavoha (A), hb-ga dans les métatroctolites (-) et dans les amphibolites et anorthosite d'Anavoha ( $\bullet)$; lignes : $\mathbf{K d}=1$ et 3.

Magnesium-iron distribution for the mineral pairs : opx-cpx $(+)$, opx-hb $(*)$ in metatroctolites, hb-gd (o) in corundum amphibolites and anorthosite from Anavoha (A), hb-ga in metatroctolite ( $(-)$ and in conundum amphibolites and anorthosite from Anavoha (๑) ; lines : $K d=1$ and 3.

$4,9 \%$ et $7,4 \%$ respectivement. Selon ce dernier auteur, c'est le chrome qui, se substituant à l'aluminium, stabilise les saphirines peralumineuses. Le coefficient de répartition $\mathrm{Fe}-\mathrm{Mg}$ entre saphirine et spinelle vert (Figure 8) est voisin de 0,28 , conformément à la composition de Higgins et al. (1979). Par contre, le Kd entre saphirine et spinelle brun chromifère s'écarte sensiblement de cette valeur: il est égal à 0,14 dans une amphibolite (éch. 54b) et 0,19 dans l'anorthosite. Braud (1981) remarque également une dispersion des couples de minéraux chromifères par rapport à la valeur de $\mathbf{0 , 2 8}$. En effet, dans le spinelle, la substitution de l'aluminium par le chrome s'accompagne de la substitution du magnésium par le fer. Ainsi, le coefficient de distribution varie en fonction de la teneur en chrome dans le spinelle et il semble qu'il existe une relation linéaire entre $1 n \mathrm{Kd}$ et le rapport $\mathrm{Cr}$ / $\mathrm{Cr}+\mathrm{Al}$ dans le minéral.

\section{Staurotide}

L'association de la staurotide avec des minéraux aussi magnésiens est exceptionnelle. Aussi présente-t-elle des teneurs inhabituellement élevées en magnésium : entre $4,7 \%$ et $5,46 \%$ $\mathrm{MgO}$ (Tableau I) ; les valeurs du rapport $\mathrm{XMg}$ qui varient habituellement entre 14 et 39 (Gang'lly, 1972) sont ici de 44 à 52 . La formule

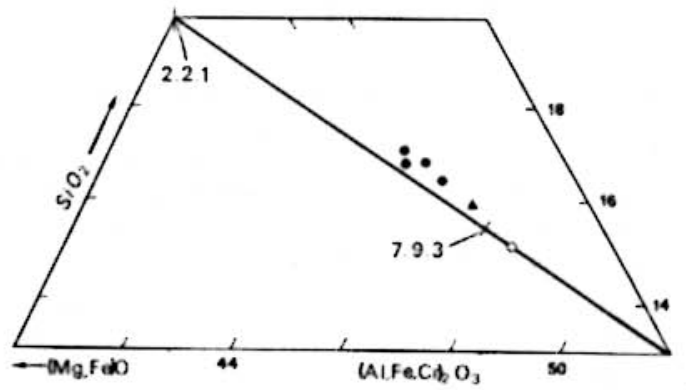

FiG. 7. - Position des saphirines des amphibolites

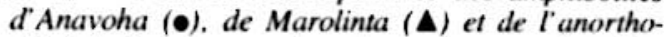
site (o) dans une portion du système ternaire $(\mathrm{Mg}$. $\mathrm{Fe}) \mathrm{O}-(\mathrm{Al}, \mathrm{Fe}, \mathrm{Cr})_{2} \mathrm{O}_{3}-\mathrm{SiO}_{2}$ (Higgins et al., 1979).

Sapphirines of amphibolites from Anavoha (๑). Marolinta $(\Delta)$ and anorthosite $(o)$ plotted in a part of the ternary system $(\mathrm{Mg}, \mathrm{Fe}) \mathrm{O}-(\mathrm{Al}, \mathrm{Fe}, \mathrm{Cr})_{2} \mathrm{O}_{3}-\mathrm{SiO}_{2}$ (Higgins et al., 1979).

structurale est calculée sur la base de $\mathbf{4 6}$ oxygènes, équivalant à $44(\mathrm{O})+4(\mathrm{OH})$, comme le suggèrent Griffen et al., (1982). Si varie entre 7,52 et 7,78. Ces valeurs sont élevées par rapport aux staurotides magnésiennes de la littérature : 7,09. 7,28 (Grew et Sandiford, 1984), 7,23 (Schreyer et al., 1984) ; 7,37-7,54 (Ward, 1984). Les teneurs en titane $(0,24$ à $1,08 \%)$ et chrome $(0,8$ à $2,2 \%)$ sont relativement fortes. Ce dernier élément est certainement responsable de la teinte verte selon $\mathrm{Nm}$, comme le suggère Ward (1984). Le manganèse n'a pas été détecté ; le zinc n'a pas été analysé. Les compositions de la staurotide se situent largement en dessous de la "ligne de probabilité maximale" de Griffen et al., (1982): $\mathrm{Al}+\mathrm{Si}-8=\mathrm{Al}^{\prime}=20,5-0,80(\mathrm{Fe}+$

TABLEAU I. - Analyses des minéraux des amphibolites à corindon de Marolinta (éch. 50b) et d'Anavoha (éch. $54 \mathrm{~b}$ et A7) et de l'anorthosite (éch. A24) ; ( ) : paragenèse : voir texte. Analyses réalisées par D. Ackermand (Kiel).

Analyses of minerals of curundum amphibolites from Marolinta (sample 50b) and Anavoha (samples 54b, A7) and of the anorthosite from Anavoha (sample A24) ; ( ) : paragenesis : see text. 


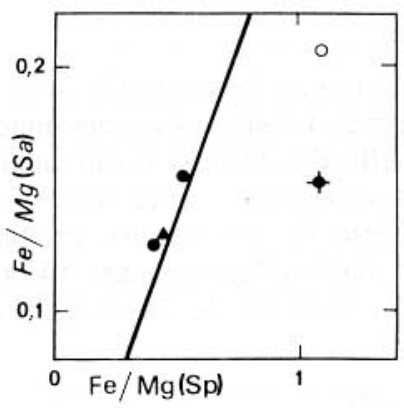

FIG. 8. - Répartition fer - magnésium dans les couples spinelle vert - saphirine dans les amphibolites de Marolinta $(\boldsymbol{\Delta})$ et d'Anavoha $(\bullet)$ et les couples spinelle brun chromifère - saphirine dans l'amphibolite $54 \mathrm{~b}$ (†) et dans l' anorthosite $(o)$; ligne : $\mathrm{Kd}=$ 0,28 , d'après Higgins et al, (1979).

Iron - magnesium distribution in green spinel sapphirine pairs in amphibolites from Marolinta $(\mathbf{\Delta})$, Anavoha (•) and in brown Cr-spinel - sapphirine pairs in amphibolite $54 b(\$)$ and anorthosite $(o)$; line : $\mathrm{Kd}=0,28$, after Higgins et al. (1979).

$\mathrm{Mg}+\mathrm{Zn}$ ) ou celle de Ward (1984) calculée par Grew et Sandiford (1985): $\mathrm{Al}^{\prime}=19,9-0,63$ $(\mathrm{Mg}+\mathrm{Fe})$ (Figure 9). L'écart entre les analyses et ces lignes varie entre 0,05 et 0,55 atomes et est étroitement lié à la teneur en chrome du minéral. Aussi, si l'on ajoute $\mathrm{Ti}$, comme le suggèrent Schreyer et al., (1984) et $\mathrm{Cr}$ à $\mathrm{Al}^{\prime}$, les staurotides d'Anavoha vont se regrouper autour de ces lignes. Il en est de même pour les staurotides chromifères de Fiordland (Ward, 1984) (Figure 9). Ceci confirme la substitution de l'aluminium octaédrique par le chrome suggérée par Ward (1984).

Le $\mathrm{Kd} \mathrm{Fe} / \mathrm{Mg}$ entre le grenat et la staurotide est supérieur à l'unité $(\mathrm{Kd}=1,14$ à 1,54$)$, ce qui représente une distribution "normale" (Holdaway, 1978) qui peut s'expliquer par le fait que ces deux minéraux sont en équilibre textural. Le coefficient de distribution du fer et du magnésium entre la staurotide et le spinelle brun est voisin de l'unité.

\section{Autres minéraux :}

Un peu de chrome est à signaler dans le disthène $(\leqslant 0,9 \%)$ et le corindon $(\leqslant 0,75 \%)$. Ce dernier minéral peut contenir à la place un peu de fer $(=0,5 \%)$. Le titane n'y a pas été détecté. La chlorite de l'anorthosite est très magnésienne (Tableau I).

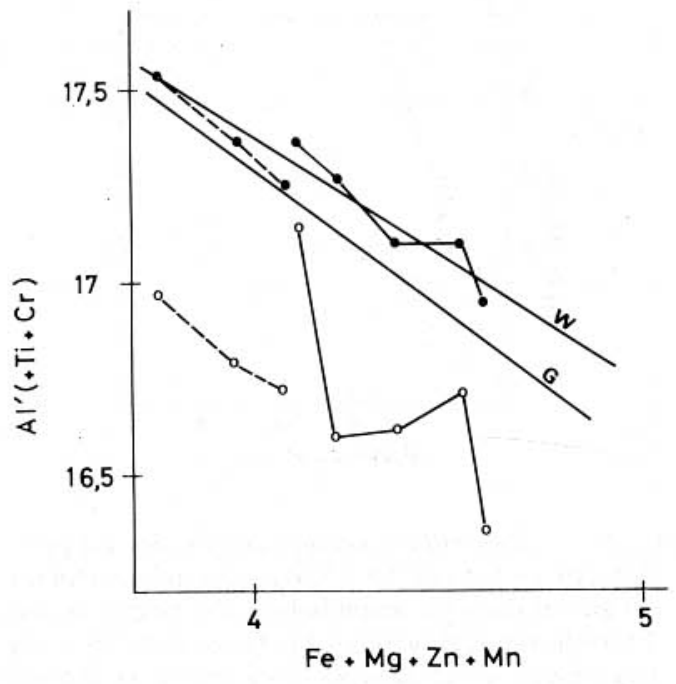

FIG. 9. - $A l(=A l+S i-8)$ (o) et $A l^{\prime}+T i+C r(\bullet)$ versus $\mathrm{Mg}+\mathrm{Fe}+\mathrm{Mn}+\mathrm{Zn}$ pour les staurotides de l'anorthosite d'Anavoha (ligne pleine) et pour les staurotides chromifères de Fiordland (ligne pointillé ; Ward, 1984). "Lignes de probalité maximale" de Griffen et al., 1982:(G) et de Ward, 1984, calculée par Grew et Sandiford, 1985 : (W).

$A l^{\prime}(=A l+S i-8) \quad(o)$ and $A l^{\prime}+T i+C r$ (•) versus $\mathrm{Mg}+\mathrm{Fe}+\mathrm{Mn}+\mathrm{Zn}$ for staurolites from the Anavoha anorthosite (solid line) and for chromian staurolites from Fiordland (dashed line; Ward, 1984). "'Maximum likelihood lines"' of Griffen et al., 1982 : (G) and of Ward, 1984, calculated by Grew and Sandiford, $1985:(\mathrm{W})$.

\section{ORIGINE DES AMPHIBOLITES À CORINDON - SAPHIRINE OU GÉDRITE ET ROCHES ASSOCIÉES}

Des roches similaires sont rencontrées dans de nombreux pays: les amphibolites à saphirine sont signalées en Caroline du Nord (Hadley, 1949) ; dans le Massif Central français (Forestier et Lasnier, 1969); elles sont associées à des roches ultrabasiques à spinelle en Finlande (Haapala et al., 1971), localisées au contact gabbro-lherzolite dans la zone d'Ivrée (Sills et al., 1983) ; au Groenland, les assemblages à saphirine apparaissent au contact supérieur du complexe anorthositique de Fiskenaesset (Herd, 1973 ; Friend, 1982) ; dans le massif d'anorthosite de Sittampundi, en Inde, la saphirine se trouve dans des niveaux anorthositiques séparés par des niveaux de chromitite (Janardhan et Leake, 1974 ; Grew, 1982). Une origine ortho- 
dérivée est toujours retenue par les auteurs. Cependant, la formation de saphirine peut être liée à un échange de matériel entre anorthosite et chromitite (Grew, 1982) ou entre anorthosite et roches ultrabasiques (Friend, 1982).

La staurotide magnésienne est un minéral rare rencontré dans le même type de roche que la saphirine : métasédiments (Grew et Sandiford, 1984 ; Schreyer et al., 1984) et métabasites (éclogite : Smith, 1984 ; métatroctolite : Ward, 1984) ; ces roches sont peralumineuses, avec un rapport $\mathrm{Mg} / \mathrm{Fe}$ élevé, et pauvres en silice. Gibson (1978) observe une staurotide de composition "normale" $(\mathrm{XMg} \simeq 30)$ dans des amphibolites à disthène ou corindon.

A Anavoha, le passage entre les métatroctolites et les amphibolites à corindon suggère que l'ensemble de ces roches provient de la transformation métamorphique d'un massif de troctolite. Les analyses chimiques (Tableau II) d'une métatroctolite, d'une amphibolite d'Anavoha (paragenèse 4) et d'une amphibolite de Marolinta (paragenèse 2) confirment cette hypothèse. Les trois roches ont des compositions chimiques très voisines, à l'exception de l'eau. L'amphibolite de Marolinta a une teneur en $\mathrm{H}_{2} \mathrm{O}$ particulièrement élevée ainsi que $0,83 \%$ de $\mathrm{K}_{2} \mathrm{O}$. Ceci est certainement lié à des circulations hydrothermales matérialisées par la transformation du plagioclase en muscovite et par l'existence de chlorite dans les fissures. La composition minéralogique normative fait apparaître dans les trois roches environ $60 \%$ de plagioclase et $30 \%$ d'olivine. Ces roches ont des compositions de leucotroctolites, comme les roches à saphirine de la Zone d'Ivrée (Sills et al., 1983) et celle du Massif Central français (Lasnier, 1977). Le passage progressif de l'amphibolite à l'anorthosite avec une variation de la proportion plagioclase/ amphiboles témoigne d'une variation dans la composition minéralogique de la roche magmatique initiale. Ainsi, serpentinites, métatroctolites, amphibolites et anorthosites sont le produit de la transformation métamorphique d'un ancien complexe magmatique cumulatif stratifié contrôlé par le fractionnement à basse pression d'olivine + plagioclase + clinopyroxène. L'abondance de la saphirine dans les amphibolites n'est pas influencée par la proximité du filon d'anorthosite ; ceci exclut la formation de ce minéral par échange métasomatique entre métabasite et anorthosite.

\begin{tabular}{|c|c|c|c|c|c|c|c|}
\hline & 24 & 52 & $50 b$ & & $A 4$ & 52 & 500 \\
\hline $510_{2}$ & 44,19 & 42,96 & $43,8 I$ & & & & \\
\hline $\mathrm{Al}_{2} \mathrm{O}_{3}$ & $2 \mathrm{I}, 42$ & 21,80 & 20,60 & & & & \\
\hline $\mathrm{F}_{2} \mathrm{O}_{3}$ & 7,40 & 6,23 & 4,80 & 01 & 30,2 & 29,7 & 30,4 \\
\hline mno & 0,11 & 0,10 & 0,09 & $\mathrm{Hy}$ & 2,2 & - & - \\
\hline ngo & 13,07 & 13,31 & 14,33 & DI & 1,4 & 4,4 & 2,3 \\
\hline CaO & 10,87 & 11,90 & 10,12 & or & 0,3 & 0,4 & 5 \\
\hline $\mathrm{Na}_{2}{ }_{2}$ & 1,37 & $\mathrm{I}, 2 \mathrm{II}$ & 1,37 & AD & 11,5 & 6,3 & 11,5 \\
\hline$k_{2}{ }^{0}$ & 0,05 & 0,07 & 0,83 & an & 52,3 & 53,7 & 47,5 \\
\hline $\mathrm{TiO}_{2}$ & $0,1 \theta$ & 0, II & 0,13 & Ne & - & 2,3 & - \\
\hline $\mathrm{P}_{2} \mathrm{O}_{5}$ & 0,05 & 0,02 & 0,02 & I1n & 0,3 & 0,2 & 0,2 \\
\hline $\mathrm{H}_{2} \mathrm{O}$ & 0,42 & 1,37 & 3,07 & $A p$ & $0, I$ & $0, I$ & 0,1 \\
\hline Tota1 & 99,13 & 99,07 & 99,17 & $\frac{A n}{A n A B}$ & 82 & 89,5 & 80,5 \\
\hline$\times \mathrm{mg}$ & 78 & $\theta \mathrm{I}$ & 86 & & & & \\
\hline
\end{tabular}

TABLEAU II. - Analyses d'une métatroctolite (A4), d'une amphibolite à saphirine-corindon-grenat (52) d'Anavoha et d'une amphibolite à corindon-gédrite (50b) de Marolinta. Analyses réalisées au Centre Géologie Géophysique de I'USTL Montpellier.

Chemical compositions of a metatroctolite (A4), a saphirine - corundum - garnet amphibolite (52) from Anavoha and a corundum - gedrite amphibolite (50b) from Marolinta.

\section{EVOLUTION MÉTAMORPHIQUE DU COMPLEXE MAGMATIQUE}

Les métatroctolites et amphibolites à corindon, saphirine ou gédrite peuvent s'être formées au cours d'un même épisode métamorphique ou bien au cours de deux épisodes successifs, les amphibolites résultant de la rétromorphose des métatroctolites. Les variations linéaires du rapport $\mathrm{XMg}$ (Figure 6), identiques entre les couples hb-gd dans les amphibolites et opx-hb dans les métatroctolites d'une part, et dans les couples hb-ga dans les deux types de roches, suggèrent que les différents assemblages minéralogiques sont à l'équilibre et qu'ils se sont formés dans des conditions Pt-T identiques, au cours d'un seul épisode métamorphique. En l'absence de dosage du fluor dans les amphiboles, on peut penserque seule l'activité de l'eau aurait varié, croissante depuis les assemblages coronitiques à deux pyroxènes puis à un pyroxène, avec $\mathrm{PH}_{2} \mathrm{O} \ll \mathrm{Pt}$, jusqu'aux amphibolites dans lesquelles la pression d'eau aurait approché la pression totale. Ceci nécessite une variation brutale de l'apport d'eau à l'intérieur du massif de troctolite. A moins que la petite quantité d'eau nécessaire à la coronitisation ne soit déjà présente dans la structure des minéraux de la roche magmatique, comme le suggère Barink (1984) dans un métagabbro. Dans ce cas, seule 
l'amphibolitisation nécessiterait un apport d'eau de l'extérieur. La disparition de la saphirine puis du corindon au profit du spinelle vert sombre dans les amphibolites à gros grain, sans gédrite, peut correspondre à une évolution rétrograde dans les conditions du faciès amphibolite (Lasnier, 1977).

\section{Coronitisation de la troctolite :}

La présence d'olivine au cœur des amas d'orthopyroxène montre que les ensembles coronitiques résultent de réactions classiquement décrites entre l'olivine et le plagioclase sous les conditions du faciès granulite (Griffin et Heier, 1973). La présence de phases minérales différentes dans les amas coronitiques d'un même échantillon montre que des équilibres très localisés peuvent exister, certainement grâce à une faible mobilité des phases fluides. De nombreuses réactions subsolidus ont été proposées pour expliquer la formation de couronnes multiples : ces réactions impliquent généralement une évolution rétrograde par refroidissement d'un magma basique en climat métamorphique.

\section{Amphibolites à saphirine ou gédrite :}

Les amphibolites du Vohibory et les roches à saphirine orthodérivées de la littérature se distinguent des roches à saphirine aérivant de métasédiments magnésiens par la présence d'une proportion notable de calcium. De ce fait, les roches orthodérivées et leurs minéraux doivent être représentés dans un modèle simplifié par un minimum de cinq constituants chimiques : $\mathrm{SiO}_{2}-\mathrm{Al}_{2} \mathrm{O}_{3}-\mathrm{MgO}-\mathrm{FeO}-\mathrm{CaO}\left(-\mathrm{H}_{2} \mathrm{O}\right.$ en excès). On néglige le sodium en faible quantité dans le plagioclase et la hornblende ainsi que le chrome principalement concentré dans le spinelle brun, rare. Si nous traitons $(\mathrm{Mg}, \mathrm{Fe}) \mathrm{O}$ comme un seul constituant, nous pouvons représenter minéraux et roches dans un tétraèdre $\mathrm{SiO}_{2}-\mathrm{Al}_{2} \mathrm{O}_{3}-(\mathrm{Mg}, \mathrm{Fe}) \mathrm{O}-$ $\mathrm{CaO}$ et utiliser une projection sur le plan $\mathrm{SiO}_{2}-$ $\mathrm{Al}_{2} \mathrm{O}_{3}-(\mathrm{Mg}, \mathrm{Fe}) \mathrm{O}$ à partir de l'anorthite (Figure 10 ). Les paragenèses à grenat d'Anavoha (Figures $10 a$ et $b$ ) contiennent cinq phases minérales, ce qui suggère que $\mathrm{MgO}$ et $\mathrm{FeO}$ doivent être traités séparément, comme l'ont déjà remarqué Lal et al. (1978). Toutefois, la figure $10 c$ montre clairement que la présence de saphirine $o u$ de gédrite est liée à des variations du rapport $\mathrm{SiO}_{2} /(\mathrm{Mg}, \mathrm{Fe}) \mathrm{O}$ de la roche. Ces variations sont réalisées par une diminution de la proportion $\mathrm{Ol} / \mathrm{Pl}$ ou par une baisse de la teneur en anorthite du plagioclase (Tableau II) dans la roche magmatique initiale. L'association $\mathrm{hb}+\mathrm{co}$ exclut l'association $\mathrm{gd}+\mathrm{sa}$ dans les roches à plagioclase selon la réaction $\mathrm{hb}+\mathrm{co} \rightleftharpoons \mathrm{gd}+\mathrm{sa}+\mathrm{pl}+\mathrm{v}$. L'existence de saphirine rare dans l'amphibolite 54b, à deux amphiboles et grenat, est peutêtre le témoin de cette réaction (Figure $10 b$ ) qui est en fait divariante si l'on considère $\mathrm{Fe}$ et $\mathrm{Mg}$. Janardhan et Leake (1974) mentionnent également l'assemblage clinoamphibole $+\mathrm{gd}+\mathrm{pl}+\mathrm{co}$ + sa dans le massif de Sittampundi.

La majorité des paragenèses contient du corindon : l'alumine est en excès. Nous pouvons donc utiliser le tétraèdre $\mathrm{SiO}_{2}-\mathrm{MgO}-\mathrm{FeO}-\mathrm{CaO}$ en projetant sur le plan $\mathrm{SiO}_{2}-\mathrm{MgO}-\mathrm{FeO}$ à partir de l'anorthite (Figure 11 ). La figure $11 b$ résume les paragenèses des amphibolites à corindon. Il apparaît nettement que les minéraux et roches totales sans grenat de Marolinta sont plus magnésiens que les minéraux et roches à grenat d'Anavoha.

\section{L'anorthosite à corindon - grenat - staurotide magnésienne - saphirine :}

La figure $11 a$ représente les associations minérales observées dans l'anorthosite d'Anavoha. Certaines lignes de liaison se coupent alors que les associations minérales - en particulier gdhb-ga et std-ga-sa - paraissent à l'équilibre. Ces intersections peuvent s'expliquer par le fait que nous prenons en considération des phases en très petite quantité (saphirine, spinelle brun) et qu'il est donc nécessaire de faire intervenir également des composants chimiques en faible proportion : il s'agit du sodium présent dans les amphiboles et le plagioclase et du chrome présent dans tous les minéraux ferromagnésiens à l'exception du grenat. Les assemblages à deux amphiboles $(+$ corindon) semblent se former préférentiellement aux assemblages à std-sa (+ anorthite) en présence de sodium, ce qui expliquerait la rareté de std-sa et la fréquence de gd-hb dans la nature ainsi que les variations en sens opposé des proportions de staurotide et d'amphibole dans l'anorthosite à corindongrenat. Cependant, il est également possible que nous ayons des équilibres localisés correspondant à des conditions différentes de $\mathrm{P}_{\mathrm{H}_{2} \mathrm{O}}$.

L'association staurotide - disthène à l'intérieur du grenat suggère que la staurotide ait pu se former au cours d'une réaction similaire à celle invoquée par Ganguly (1972) pour la Fe staurotide : 


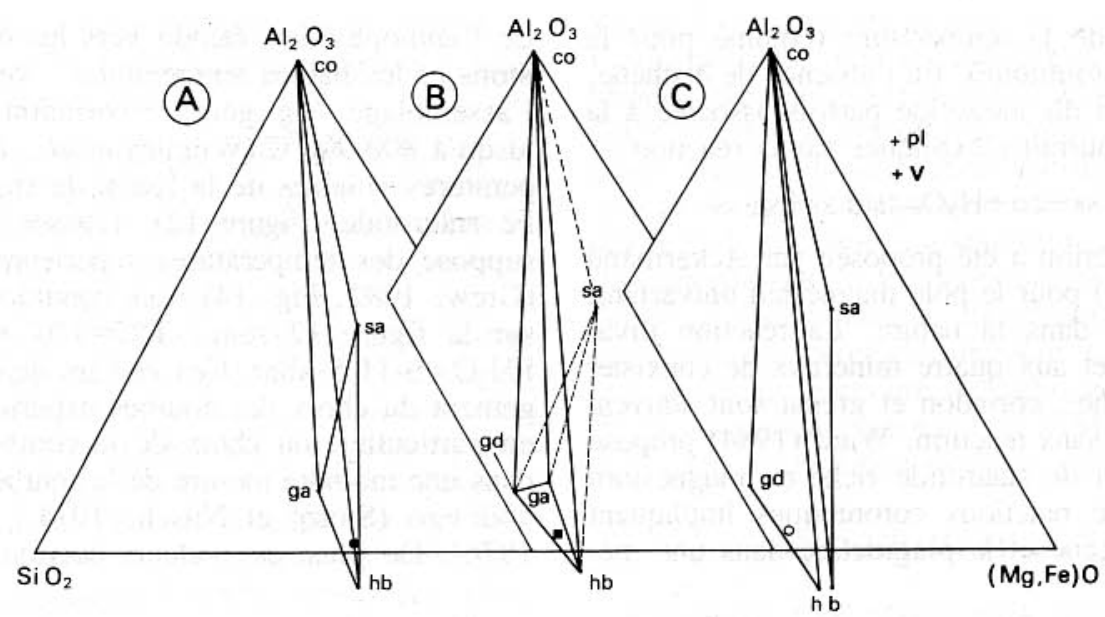

FIG. 10. - Projection à partir de l'anorthite sur le plan $\mathrm{SiO}_{2}-\mathrm{Al}_{2} \mathrm{O}_{3}-(\mathrm{Mg}, \mathrm{Fe}) \mathrm{O}$ du système $\mathrm{SiO}_{2}-\mathrm{Al}_{2} \mathrm{O}_{3}-(\mathrm{Mg}, \mathrm{Fe}) \mathrm{O}$ $\mathrm{CaO}-\left(\mathrm{H}_{2} \mathrm{O}\right)$. Anavoha : A) Echantillon 52 (minéraux et roche totale : •) ; B) Echantillons 54 b (minéraux) et métatroctolite AI (roche totale : - ) ; lignes pointillées : assemblage relictuel (?) Marolinta : C) Echantillons $50 \mathrm{a}$ (minéraux) et $50 \mathrm{~b}$ (minéraux et roche totale : o).

Projection from anorthite onto the $\mathrm{SiO}_{2}-\mathrm{Al}_{2} \mathrm{O}_{3}-(\mathrm{Mg}, \mathrm{Fe}) \mathrm{O}$ plane in the system $\mathrm{SiO}_{2}-\mathrm{Al}_{2} \mathrm{O}_{3}-(\mathrm{Mg}, \mathrm{Fe}) \mathrm{O}-\mathrm{CaO}$ $\left(\mathrm{H}_{2} \mathrm{O}\right)$. Anavoha : A) sample 52 (minrals and rock: $\bullet$ ) ; B) samples $54 \mathrm{~b}$ (minerals) and metatroctolite AI (whole rock : —) ; dashed lines : relict assemblage (?) Marolinta : C) samples 50a (minerals) and 50b (minerals and whole rocke : o).

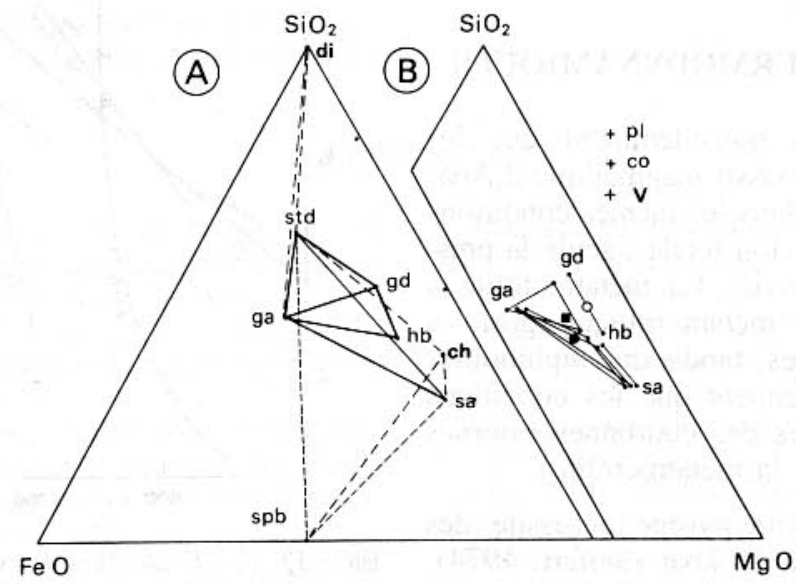

FIG. 11. - Projection à partir de l'anorthite sur le plan $\mathrm{SiO}_{2}-\mathrm{FeO}-\mathrm{MgO}$ dans le système $\mathrm{SiO}_{2}-\mathrm{Al}_{2} \mathrm{O}_{3}-\mathrm{MgO}-\mathrm{FeO}$ $\mathrm{CaO}-\left(\mathrm{H}_{2} \mathrm{O}\right) . \mathrm{Al}_{2} \mathrm{O}_{3}$ et $\mathrm{H}_{2} \mathrm{O}$ sont en excès. A) Anorthosite d'Anavoha ; lignes pointillées : réactions possibles d'apparition et disparition de la staurotide ; B) Amphibolites (minéraux : Anavoha : 54b, A7, A9 ; Marolinta : $50 \mathrm{a}, 50 \mathrm{~b}$; roches : voir figure 10 ).

Projection from anorthite onto the $\mathrm{SiO}_{2}-\mathrm{FeO}-\mathrm{MgO}$ plane in the system $\mathrm{SiO}_{2}-\mathrm{Al}_{2} \mathrm{O}_{3}-\mathrm{MgO}-\mathrm{FeO}-\mathrm{CaO}-\left(\mathrm{H}_{2} \mathrm{O}\right)$ with excess of $\mathrm{Al}_{2} \mathrm{O}_{3}$ and $\mathrm{H}_{2} \mathrm{O}$. A) Anorthosite from Anavoha, dashed lines : possible formation and breakdown reactions of staurolite ; B) Amphibolites (minerals : 54b, A7, A9, Anavoha, 50a, 50b Marolinta ; whole rocks : see figure 10 ). 
par baisse de la température (comme pour la troctolite coronitique). En l'absence de disthène, la formation de staurotide parfois associée à la saphirine pourrait s'expliquer par la réaction :

$$
\text { ga ss }+\mathrm{co}+\mathrm{H}_{2} \mathrm{O} \rightleftharpoons \text { std ss }+ \text { sa ss }
$$

Cette réaction a été proposée par Ackermand et al. (1975) pour le pôle magnésien univariant, non réalisé dans la nature. La réaction divariante permet aux quatre minéraux de coexister dans la roche : corindon et grenat sont souvent en contact, sans réaction. Ward (1984) propose la formation de staurotide riche en magnésium au cours de réactions coronitiques impliquant l'orthopyroxène et le plagioclase dans une métatroctolite.

Le spinelle brun est associé à la staurotide et à la saphirine (Figure 5). Cette association peut être liée à une syncristallisation ou bien à un début de déstabilisation de la staurotide. Dans ce dernier cas, la chlorite parfois présente pourrait participer à cette déstabilisation et on peut proposer la réaction :

$\mathrm{std} \pm \mathrm{sa}+\mathrm{pl} 1 \pm \mathrm{H}_{2} \mathrm{O} \rightleftharpoons \mathrm{spb}+\mathrm{pl} 2 \pm \mathrm{Ch}$ (Figure 11a)

\section{CONDITIONS THERMODYNAMIQUES}

Nous avons admis précédemment que les différentes roches du massif magmatique d'Anavoha se sont formées dans les mêmes conditions de température et pression totale : seule la pression partielle d'eau varie. La métatroctolite a enregistré l'évolution métamorphique grâce à ces couronnes minérales, tandis qu'amphibolites et anorthosite ne retiennent que les conditions finales (contemporaines des couronnes externes à pargasite + grenat de la métatroctolite) .

L'association saphirine-pyrope nécessite des pressions supérieures à 15 kbar (Seifert, 1974). Celles-ci sont notablement diminuées par la présence des composants almandin (Hensen et Green, 1971) et grossulaire. Toutefois, le grenat des amphibolites reste riche en composant pyrope (jusqu'à $60 \%$ ), ce qui suppose des pressions relativement élevées. Les conditions P-T des amphibolites à saphirine et roches associées peuvent être estimées grâce à la présence dans l'anorthosite d'Anavoha de saphirine, de disthène, de gédrite et de l'assemblage anorthite+ corindon (Figure 12). Notons que la gédrite possède un domaine de stabilité plus vaste que celui de l'anthophyllite, étendu vers les hautes pressions et les hautes températures : vers $10 \mathrm{kbar}$, l'assemblage $\mathrm{Mg}$ gédrite+corindon est stable jusqu'à $800-900^{\circ} \mathrm{C}$ (Windley et al., 1984), températures voisines de la limite de stabilité de la $\mathrm{Fe}$ staurotide (Figure 12). L'association ga-sa suppose des températures supérieures à $730^{\circ} \mathrm{C}$ (Grew, 1982, Fig. 14). Les conditions définies sur la figure 12 sont : $\mathrm{T}^{\circ} \mathrm{C}=750^{\circ}-850^{\circ} \mathrm{C}$ pour $\mathrm{PH}_{2} \mathrm{O}=9-11,5$ kbar. Ces valeurs dépendent largement du choix des courbes expérimentales ; en particulier, du choix de la courbe $s i \rightleftharpoons$ di et dans une moindre mesure de la courbe co+an $+\mathrm{v}$ $\rightleftharpoons$ di+zo (Storre et Nitsch, 1974 ; Chatterjee, 1976). De plus, ces valeurs doivent être nuan-

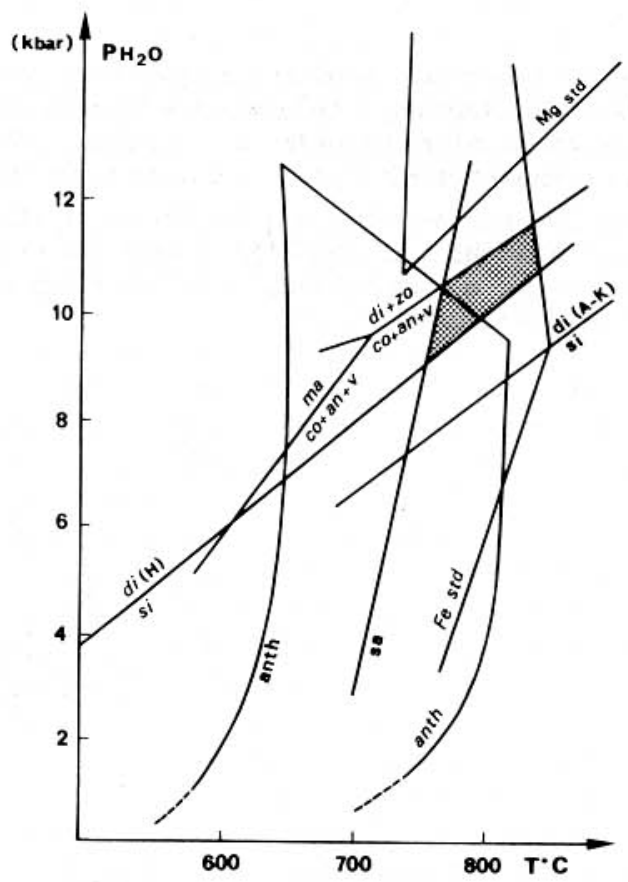

FIG. 12. - Evaluation Pression - Température des conditions de formation des amphiboles à corindon (et roches associées) à partir des courbes expérimentales de Holdaway (1971) (H) et Anderson et Kleppa (1969) $(\mathrm{A}-\mathrm{K})(\mathrm{di}=\mathrm{si})$, Storre et Nitsch, $1974(\mathrm{ma}=\mathrm{co}+\mathrm{an}+\mathrm{V} ; \mathrm{di}+\mathrm{zo}=\mathrm{co}+\mathrm{an}+\mathrm{V})$, Day et Halbach, 1979 (domaine de stabilité calculé pour l'anthophyllite), Ackermand et al., 1975 (limite de stabilité de la saphirine), Ganguly, 1972 et Schreyer, 1968 (limites de stabilité de la Fe std et $\mathrm{Mg}$ std respectivement).

$P-T$ estimates for the formation of corundum amphibolites and related rocks using experimental curves. 
cées en fonction de l'activité de $\mathrm{H}_{2} \mathrm{O}$ et de la teneur en fer des ferromagnésiens, ce qui conduit à des valeurs inférieures.

Nous avons appliqué aux amphibolites le géothermomètre hb-ga mis au point par Graham et Powell (1984). Les températures obtenues varient entre $640^{\circ} \mathrm{C}$ et $730^{\circ} \mathrm{C}$. Les températures inférieures à $700^{\circ} \mathrm{C}$ sont incompatibles avec la présence de la saphirine (si l'on considère que $\mathrm{Pt}$ est voisine de $\mathrm{PH}_{2} \mathrm{O}$ ) et avec l'association sa-ga. La paragenèse des granulites à grenat associées aux amphibolites d'Anavoha est : salite + grenat alm+magnésiohornblende brune+andésine \pm ilménite. La température obtenue grâce au couple clinopyroxène - grenat varie de $750^{\circ} \mathrm{C}$ (bordure des minéraux) à $800^{\circ} \mathrm{C}$ (cœur des minéraux) en utilisant la calibration d'Ellis et Green (1979) qui prend en compte l'influence du composant grossulaire du grenat. Le couple hb - ga donne une température de $740^{\circ} \mathrm{C}$. L'ensemble de ces données permet d'estimer les conditions de formation des associations à saphirine et staurotide entre 750 et $800^{\circ} \mathrm{C}, 9$ et $11,5 \mathrm{kbar}$.

En Afrique du Sud, Schreyer et al. (1984) signale une staurotide magnésienne, relictuelle à 7-8 kbar pour une température de $800^{\circ} \mathrm{C}$ : selon ces auteurs, cette staurotide aurait été stable à plus haute pression et plus basse température. En Antarctique, une staurotide magnésienne est blindée dans un assemblage formé à $6 \mathrm{kbar}-$ $700^{\circ} \mathrm{C}$ (Grew et Sandiford, 1984) ; cet assemblage succède à un assemblage dans lequel la staurotide était à l'équilibre, dans le champ de stabilité du disthène. La staurotide magnésienne de l'anorthosite du Vohibory semble montrer un début de déstabilisation. En conséquence, nous pouvons considérer en première approximation que la limite inférieure de stabilité en pression de la staurotide magnésienne $(X M g \simeq 50)$ coïncide avec la transition disthène-sillimanite.

\section{REMERCIEMENTS}

Ce travail a été réalisé avec l'autorisation du Ministère de la Recherche Scientifique de Madagascar et grâce à l'aide matérielle du Laboratoire de Pétrologie des Zones Profondes de Montpellier et de D. Ackermand de l'Institut de Minéralogie de l'Université de Kiel. La plupart des dessins sont de M. Bœuf. Je remercie E.S. Grew, F. Seifert et C. Chopin pour leurs suggestions et commentaires.

Reçu le 9 septembre 1985 Accepté le 10 avril 1986

\section{REFERENCES}

ACKermand, D., Seifert, F. et Schreyer, N. (1975). - Instability of sapphirine at high pressures. Contrib. Mineral. Petrol., 50, 79-92.

ANDERSON, P.A.M. et KLEPPA, O.J. (1969). - The thermochemistry of kyanite-sillimanite equilibrium. Am. J. Sci., 267, 285-290.

BARINK, H.W. (1984). - Replacement of pyroxene by homblende, isochemically balanced with replacement of plagioclase by garnet, in a metagabbro of upper-amphibolite grade. Lithos, 17, 247-258.

Boulanger, J. (1954). - Etude géologique des schistes cristallins des feuilles Sakoa, Sakamena, Ianapera, Benenitra. TBG 56 Tananarive.

BOULANGER, J. (1957). - Le système volcanique fissural du pays Mahafaly (SW de Madagascar). Bull. Soc. Géol. Fr., 6, 529-537.

BRAUD, C. (1981). - Origine et signification de la saphirine et des minéraux associés dans les amphibolites à saphirine et corindon de la série du Haut Allier. (Massif Central français). D.E.A., Univ. Montpellier (non publié).

ChATTERJEE, N.D. (1976). - Margarite stability and compatibility relations in the system $\mathrm{CaO}-\mathrm{Al}_{2} \mathrm{O}_{3}$ $\mathrm{SiO}_{2}-\mathrm{H}_{2} \mathrm{O}$ as a pressure-temperature indicator. $\mathrm{Am}$.
Mineral., 61, 699-709.

DaY, H.W. et HaLbach, H. (1979). - The stability field of anthophyllite : the effect of experimental uncertainty on permissible phase diagram topologies. Am. Mineral., 64, 809-823.

DroOP, G.T.R. et BUCHER-NURMINEN, K. (1984). Reaction textures and metamorphic evolution of sapphirine-bearing granulites from the Gruf Complex, Italian Central Alps. J. Petrol., 25, 766-803.

Ellis, D.J. et Green, D.H. (1979). - An experimental study of the effect of $\mathrm{Ca}$ upon gametclinopyroxene $\mathrm{Fe}-\mathrm{Mg}$ exchange equilibria. Contrib. Mineral. Petrol., 71, 13-22.

FORESTIER, F. et LASNIER, B. (1969). - Découverte de niveaux d'amphibolites à pargasite, anorthite, corindon et saphirine dans les schistes cristallins de la vallée du Haut Allier. Contrib. Mineral. Petrol, 23, 194-235.

FRIEND, C.R.L. (1982). - $\mathrm{Al}-\mathrm{Cr}$ substitution in peraluminous sapphirines from the Bjørnesund area, Fiskenaesset region, Southem West Greenland. Mineral. Mag., 46, 323-328.

GANGULY, J. (1972). - Staurolite stability and related paregeneses: theory, experiments and appli- 
cations. J. Petrol., 62, 335-365.

GiBSON, G.M. (1978). - Staurolite in amphibolite and homblendite sheets from the upper Seaforth river, Central Fiorland, New Zealand. Mineral. Mag., 42, 153-154.

Graham, C.M. et POWELl, R. (1984). - A garnethornblende geothermometer: calibration, testing and application to the Pelona Schist, Southern $\mathrm{Ca}$ lifornia. J. metamorphic Geol., 2, 13-31.

GREW, E.S. (1982). - Sapphirine, kornerupine and sillimanite + orthopyroxène in the charnockitic region of South India. J. Geol. Soc. India, 23, 469505.

Grew, E.S. et SANDIFord, M. (1984). - A talcstaurolite assemblage in tourmaline-phlogopitechlorite schist from northern Victoria Land, Antarctica and its petrogenetic significance. Contrib. Mineral. Petrol., 87, 337-350.

GREW, E.S. et SANDIFORD, M. (1985). - Staurolite in a garnet hornblende - biotite schist from the Lanterman Range, northern Victoria Land, Antarctica. N. Jb. Miner. Mh., 9, 396-410.

GRIFFen, D.T., Gosney, T.C. et PHILliPS, W.R: (1982). - The chemical formula of natural staurolite. Am. Mineral., 67, 292-297.

GRIFFIN, W.L. et HEIER, K.S. (1973). - Petrological implications of some corona structures. Lithos, 6 , 315-335.

HaApala, I., Sitvola, J., OjanPera, P. et Yletyi-NEN, V. (1971). - Red corundum, sapphirine and kornerupine from Kittiläl, Finnish Lapland. Bull. Geol. Soc. Finland, 43, 221-231.

HadLEY, J.B. (1949). - Preliminary report on corundum deposits in the Buck Creek peridotite clay country, North Carolina. U.S. Geol. Surv. Bull., 948E, 103-128.

HENSEN, B.J. et GREEN, D.H. (1971). - Experimental study of the stability of cordierite and garnet in pelitic compositions at high pressure and temperature. I : Compositions with excess aluminosilicate. Contrib. Mineral. Petrol., 33, 309-330.

HERD, R.K. (1973). - Sapphirine and kornerupine occurrences within the Fiskenaesset complex. Gron. Geol. Unders $\phi$ gelse, 51, 65-71.

Higgins, J.B., RibBe, P.H. et HeRD, R.K. (1979). Sapphirine I : Crystal chemical contributions. Contrib. Mineral. Petrol., 68, 349-356.

HOLDAWAY, M.J. (1971). - The aluminium silicate triple point. Am. J. Sci., 269, 97-131.

HOLDAWAY, M.J. (1978): - Significance of chloritoid-bearing and staurolite-bearing rocks in the Picuris Range, New Mexico. Bull. Geol. Soc. Amer., 89, 1404-1414.

JANARDHAN, A.S. et LEAKE, B.E. (1974). - Sapphirine in the Sittampundi complex, India. Mineral. Mag., 39, 901-902.

LACROIX, A. (1929). - Sưr un schiste cristallin à saphirine de Madagascar et sur les roches à saphi- rine en général. Bull. Soc. fr. Minéral., 52, 76-84.

LAL, R.K., ACKermand, D., Seifert, F. et HALDAR, S.K. (1978). - Chemographic relationship in sapphirine-bearing rocks from Sonapahar, Assam, India. Contrib. Mineral. Petrol., 67, 169-187.

LASNIER, B. (1977). - Persistance d'une série granulitique au cour du Massif Central français. Thèse d'Etat, Nantes.

LEAKE, B.E. (1978). - The nomenclature of amphiboles. Bull. Minéral., 101, 453-467.

NiCOllet, C. (1983). - Existence de granulites de haute pression à clinopyroxène - grenat dans les formations précambriennes du Vohibory (SW de Madagascar). C.R. Acad. Sci. Paris, 297, 145-148.

Nicollet, C. (1984): - Le volcanisme dans le SudOuest de Madagascar. J. of Afr. Earth Sci., 2, 383-388.

Robinson, P., Ross, M. et JAFFE, H.W. (1971). Compositions of the anthophyllite - gedrite series, comparisons of gedrite and hornblende, and the anthophyllite - gedrite solvus. Am. Mineral., 56, 1005-1041.

SCHREYER, W. (1968). - A reconnaissance study of the system $\mathrm{MgO}-\mathrm{Al}_{2} \mathrm{O}_{3}-\mathrm{SiO}_{2}-\mathrm{H}_{2} \mathrm{O}$ at pressures between 10 and $25 \mathrm{~Kb}$. Carnegie Inst. Wash. Year Book, 66, 380-392.

SCHREYER, W. et ABRAHAM, K. (1975). - Peraluminous sapphirine as a reaction product in kyanitegedrite-talc schist from Sar e Sang, Afghanistan. Mineral. Mag., 40, 171-180.

SCHREYER, W., HORROCKS, P.C. and ABRAHAM, K. (1984). - High-magnesium staurolite in a sapphirine-garnet rock from the Limpopo belt, Southern Africa. Contrib. Mineral. Petrol., 86, 200-207.

SEIFERT, F. (1974). - Stability of sapphirine : a study of the aluminous part of the system $\mathrm{MgO}-\mathrm{Al}_{2} \mathrm{O}_{3}$ $\mathrm{SiO}_{2}-\mathrm{H}_{2}$ O. J. Geol., 82, 173-204.

Sills, J.D., ACKermand, D., Herd, R. et WinDLEY, B.F. (1983). - Bulk composition and mineral parageneses of sapphirine-bearing rocks along a gabbro-lherzolite contact at Finero, Ivrea Zone, N Italy. J. Metamorphic Geol., I, 337-351.

SMITH, D.C. (1984). - Remarques cristallochimiques et pétrogénétiques sur des minéraux inhabituels dans les éclogites de Liset et Rekvika, Norvège. $10^{e}$ R.A.S.T. Bordeaux, 511, Soc. Geol., France.

STORRE, B. et NitsCH, K.H. (1974). - Zur Stabilität von Margarit im System $\mathrm{CaO}-\mathrm{Al}_{2} \mathrm{O}_{3}-\mathrm{SiO}_{2}$ $\mathrm{H}_{2} \mathrm{O}$. Contrib. Mineral. Petrol., 43, 1-24.

WARD, C.M. (1984). - Magnesium staurolite and green chromian staurolite from Fiorland, New Zealand. Am. Mineral., 69, 531-540.

WINDLEY, B.F., ACKERMAND, D. et HERD, R.K. (1984). - Sapphirine/kornerupine - bearing rocks and crustal uplift history of the Limpopo belt, Southern Africa. Contrib. Mineral. Petrol., 86, 342358. 